\title{
Multi-Swarm Single-Objective Particle Swarm Optimization to Extract Multiple-Choice Tests
}

\author{
Tram Nguyen \\ Faculty of Information Technology \\ Nong Lam University, Ho Chi Minh City, Vietnam \\ phuongtram.itnl@gmail.com \\ Toan Bui* and Bay $\mathrm{Vo}^{\dagger}$ \\ Faculty of Information Technology \\ Ho Chi Minh City University of Technology (HUTECH) \\ Ho Chi Minh City, Vietnam \\ *bm.toan@hutech.edu.vn \\ †vd.bay@hutech.edu.vn; bagvodinh@gmail.com
}

Received 3 January 2019

Revised 6 January 2019

Accepted 16 January 2019

Published 20 February 2019

This paper proposes the use of multi-swarm method in particle swarm optimization (PSO) algorithm to generate multiple-choice tests based on assumed objective levels of difficulty. The method extracts an abundance of tests at the same time with the same levels of difficulty and approximates the difficulty-level requirement given by the users. The experimental results show that the proposed method can generate many tests from question banks satisfying predefined levels of difficulty. Additionally, the proposed method is also shown to be effective in terms of many criteria when compared with other methods such as manually extracted tests, random methods and PSO-based methods in terms of execution time, standard deviation, the number of particles per swarm and the number of swarms.

Keywords: Test questions bank; multiple-choice tests; particle swarm optimization algorithm; multi-swarm optimization; multi-objective optimization.

\section{Introduction}

Speeding up the use of science and technology to education is inevitably demanding for sustaining the development of society and addressing its cultural and educational needs. Most schools have applied e-learning a long time ago, followed by the modified

$\dagger$ Corresponding author.

This is an Open Access article published by World Scientific Publishing Company. It is distributed under the terms of the Creative Commons Attribution 4.0 (CC-BY) License. Further distribution of this work is permitted, provided the original work is properly cited. 
method for testing and application of this technology to the examination. There is a demand for improving the quality of education. Today, many researchers and society are interested in multiple-choice tests because of the quality, reliability and accuracy. Choosing the right forms of exams, especially the formation of fair exam questions at the required level of difficulty, becomes a problem that is always put forward by educators.

However, in reality, the creation of multiple-choice tests meeting the needs of the education sector is hard to handle. First, tests need to evaluate the correct characteristics of educational quality, so one has to standardize the multi-choice question banks to ensure the accuracy of the level of difficulty of questions. So far, questions in most banks exhibit subjective difficulties, and a subjective difficulty level (DL) is determined by the user. This aspect negatively impacts the quality of the test. Therefore, it becomes necessary to have a quantitative method for question difficulties. To transform the subjective difficulty level into objective difficulty, test results of students in the past are used to evaluate questions as well as question banks. Second, when the question bank has been evaluated and the questions have an objective difficulty, the next step is to extract tests. The objective difficulty of the questions should no longer be quantified at two levels (easy-difficult or basicadvanced) or three levels (easy-medium-difficult) that are different levels of difficulty depending on the composition of bank questions. This makes extracting a capability test with the required difficulty using the traditional methods difficult and it cannot be performed with the huge search space of the question bank. For example, extracting a test with hundreds of questions $(m=100)$ from a question bank that contains thousand questions $(n=1,000)$ results in a search space of $C_{n}^{m}=\frac{1000 !}{900 ! \times 100 !}$. Third, to ensure fairness between the tests, each test should be extracted from a test matrix with similar difficulty and each student should have different tests. This problem is important when extracting multiple tests with similar difficulty for multiple students at the same time.

For these reasons outlined above, we keep on studying to come up with solutions for these cases. With traditional methods such as random extraction, which most software use, it is impossible to find the solutions, and the exhaustive search method used for this problem requires very long processing time in as shown in Refs. 1 and 2. Therefore, we propose a method based on the particle swarm optimization (PSO) to solve the problem of extracting tests from the multi-choice question banks. ${ }^{3}$ Recently, PSO algorithm has been widely applied to optimization problems in which one goal-one solution with the achievements is applied in the scheduling problem, and multiple goals-multiple solutions is also applied in other areas. However, due to the diversity of situations and the environmental solutions, the problem of extracting multiple-choice questions has posed many challenges in the past decades.

This paper solves the problem of extracting multiple-choice test based on assumed objective levels of difficulty. The objective levels of difficulty assume values coming from $(0,1]$, so if we use the normal probability functions, that hardly can extract the tests that have levels of difficulty meeting the specific difficulty-level requirement. 
Hence, this paper uses particle swarm optimization for multiple objectives in continuous space problems.

The purpose of this study is to provide academicians with efficient means of generating tests with multiple-choice questions from a question bank. In this study, PSO is used to optimize predefined criteria for selecting questions from a question bank. PSO is a very useful optimization algorithm because of its versatility. This paper aims to address the issues of early convergence, extraction time and produce tests that meet the related requirements. We thus propose a suitable target function (fitness) and apply it to PSO approach to extract the items from a question bank. The experimental results show that the PSO approach is suitable for the selection of near-optimal questions from large-scale question banks according to certain criteria, namely the duration, operating boundary, stability and standard deviation.

The paper is organized as follows. Section 1 introduces this study, some related works are discussed in Sec. 2, while the proposed method is described in Sec. 3. Section 4 analyzes the experimental results of this study. Finally, Sec. 5 provides some conclusions and suggests some potential directions for future research.

\section{Literature Review}

Optimization problems that have more than one objective function are rather common in every field or area of knowledge. In such problems, the objectives to be optimized are normally in conflict with respect to each other, which means that there is no single solution for these problems. Instead, we aim to find good "tradeoff" solutions that represent the best possible compromises among the objectives.

$\mathrm{PSO}^{4,5}$ is a randomized optimization technique based on a population developed by Eberhart and Kennedy, simulated to the behavior of flocks of birds or fish swarms. Like genetic algorithm (GA), PSO seeks optimal solutions by updating generations. However, unlike GA, PSO does not have evolutionary operation, such as crossover or mutation. Eberhart and Kennedy have hypothesized the process of finding a swarm's nest by the following rules: (1) All elements in the swarm tend to move back to the nest; (2) each element memorized the closest position it has reached. Similarly, they also hypothesized the process of finding food of the swarm in a space where the swarm members knew the amount of food information and kept the closest food location that they have reached. Then, the best way to find food is to follow the lead elements, i.e. the ones in the swarm closest to the food. From that, they proposed the PSO algorithm that simulates this scenario and uses it to solve optimization problems. In PSO, every single solution is a particle in the above scenario. Each particle is characterized by two parameters: the present position position [ ] and the velocity $v$ [ ] of the particle. These are two vectors in $R^{n}(n$ is the parameter determined from the particular problem). At the same time, each particle has a fitness value, which is evaluated by the fitness function. PSO is initialized with a group of random particles (solutions) and then searches to find an optimized solution by updating generations. In every iteration, each particle is updated by following two best values. The first one 
is the best solution (fitness), it has achieved so far, and the fitness value is also stored. This value is called $P_{\text {best }}$. Another best value that is tracked by the particle swarm optimizer is the best value, obtained so far by any particle in the population. This best value is a global best and called $G_{\text {best }}$. When a particle takes part of the population as its topological neighbors, then the best value is a local best and when a particle has the best fitness value among all the particles, then the best value is a global best. At each iteration (generation), the velocity and position of each particle are updated according to the following formulas:

$$
\begin{aligned}
v[]= & v[]+c_{1} \times \operatorname{rand}() \times\left(P_{\text {best }}[]-\operatorname{position}[]\right)+c_{2} \times \operatorname{rand}() \\
& \times\left(G_{\text {best }}[]-\operatorname{position}[]\right), \operatorname{position}[]=\operatorname{position}[]+v[],
\end{aligned}
$$

where rand( ) is random in $[0,1] ; c_{1}, c_{2}$ are factors.

The problem of extracting tests from the question banks can be solved by PSO algorithm, GA or simulate algorithm (SA) such as in Refs. 1, 2, 6 and 7; authors used GA and SA to solve the problem of extracting tests, the experimental results showed that GA reached approximately $100 \%$ success rate. On the other hand, SA has a lower success rate, yet the execution time for extracting the test of GA is shorter than SA.

Besides, many researchers have shown interests in applying PSO to many fields in reality. Niu et al. proposed a multi-swarm method for PSO though master-slave method. ${ }^{8}$ The method uses two ways to solve the problem: According to the coevolutionary relationship between master swarms and slave swarms, two versions of MCPSO are proposed, namely the competitive version of MCPSO (COM-MCPSO) and the collaborative version of MCPSO (COL-MCPSO), where the master swarm enhances its particles based on an antagonistic scenario and a synergistic scenario, respectively. In the simulation studies, several benchmark functions are performed, and the performances of the proposed algorithms are compared with the standard PSO (SPSO) and its variants to demonstrate the superiority of MCPSO. Patil and Dangewar solved the multi-objective optimization. ${ }^{9}$ Multi-objective optimization is a class of problems with solutions that can be evaluated along two or more incomparable or conflicting objectives. These types of problems differ from the standard optimization problems in that the end result is not a single best solution but rather a set of alternatives, where for each member of the set, no other solution is completely better (the Pareto set). Multi-objective optimization problems occur in many different real-world domains such as automobile design and architecture. A multiobjective particle swarm optimization (MOPSO) method can be used to solve the problem of effective channel selection. In 2011, Zhou and Tan solved the problem using MOPSO. ${ }^{10}$ However, due to many fitness evaluations as well as the task of archive maintaining, the running time of MOPSO for optimizing some difficult problems may be quite long. This paper proposes a parallel MOPSO based on consumer-level Graphics Processing Units (GPUs), which, to our knowledge, is the first approach to optimizing multi-objective problems via PSO on the platform of 
GPU. Experiments on four two-objective benchmark test functions which are function $F 1$ (convex, uniform Pareto front), function $F 2$ (convex, nonuniform Pareto front), function $F 3$ (concave Pareto front) and function $F 4$ (neither purely convex nor purely concave Pareto Front) are conducted. Compared to the CPUbased sequential MOPSO, our GPU-based parallel MOPSO is much more efficient in terms of running time, and the speedups range from 3.74 times to 7.92 times. When the problem is of large scale, i.e. the dimensionality of the decision vector is large, the speedups can be bigger than 10 times. Furthermore, the experimental results show that the larger the size of the swarm is, the more nondominated solutions can be found, the higher is the quality of solutions and the bigger is the speedup. However, these works just stopped at the level of finding a solution. Recently, many researchers have shown interests in applying PSO to many fields in reality. For example, in 2015, Zhang et $a l .{ }^{11}$ have adopted PSO in eight fields that are electrical and electronics engineering automated control systems, communication theory, research, engineering, fuel and energy, medicine, chemistry and geometry. The results that came from experiments demonstrated that the PSO algorithm is effective in eight aforementioned fields when compared to other algorithms such as SA or GA. In 2017, Zou et al. proposed a new two-level hierarchical multi-swarm cooperative teachinglearning-based optimization (TLBO) variant ${ }^{12}$ called HMCTLBO to solve the global optimization problem. In HMCTLBO algorithm, all learners are randomly divided into several subswarms with equal amounts of learners at the bottom level of the hierarchy. The learners of each swarm evolve independently only in their corresponding swarm in parallel to maintaining the diversity and improving the exploration capabilities of the population. Moreover, all the best learners from each swarm compose the new swarm at the top level of the hierarchy, and each learner of the swarm evolves according to Gaussian sampling learning. Furthermore, a randomized regrouping strategy is performed, and a subspace searching strategy based on Latin hypercube sampling is introduced to maintain the diversity of the population. In 2018, Wang et al. surveyed the use of PSO algorithms from 1995 to $2016 .{ }^{13}$ The authors introduce its origin and background and carried out theoretical analysis. They discussed the ongoing research and application in algorithm structure, parameter selection, topology structure, discrete PSO algorithm, parallel PSO algorithm and multi-objective optimization PSO.

In 2018, Bui et $a l .^{3}$ proposed the use of normal particle swarm optimization to generate a test with approximating difficulties to the required levels from the users. The test is generated from question banks that consist of various questions with different difficulties. The difficulty value of each question is judged and adapted based on users via previous real-life exams. The work evaluates three approaches, which are random, GAs and PSO. The experimental result shows that PSO gives the best performance with respect to most of the criteria.

We find that all the works discussed above are not feasible to solve the problem of extracting multiple tests, so we look for an alternative approach, the multi-swarm particle swarm optimization. 


\section{Multi-Swarm Particle Swarm Optimization}

\subsection{Problem statement}

The problem of generating multiple-choice tests is defined as generating multiplechoice tests that have levels of difficulty meeting the specific difficulty-level requirement. The question banks and each question in a bank come with preassigned objective difficulty levels by the users, and the objective levels of difficulty have the continuous value domain $(0,1]$. Additionally, an objective difficulty level is assessed based on feedbacks collected from test-takers that is defined by (1).

For example, the objective difficulty level of an item can be computed as shown in (1). The issue of extracting test questions that meet the specific difficulty-level requirement $\left(\mathrm{DL}_{R}\right)$ given by the user is thus the key in this context. Let $Q=$ $\left\{Q_{1}, Q_{2}, Q_{3}, Q_{4}, Q_{5}, \ldots, Q_{n}\right\}$ be a test question bank of $n$ questions, each of which has the attributes $C_{i}$, including a question code (CQ), a code part (CP) and an objective DL. Our objective is to generate a test including $m$ questions $(m \leq n)$ $Q_{i}=\left\{Q_{i 1}, Q_{i 2}, Q_{i 3}, Q_{i 4}, Q_{i 5}, \ldots, Q_{i m}\right\} \quad\left(Q_{i j} \in Q\right)$ which satisfy $\mathrm{DL}_{R}$, i.e. $\mathrm{DL}_{R}=$ $\frac{\sum_{j=1}^{m} Q_{i j} . D L}{m}$. This is considered as the objective function used in the problem.

An objective difficulty level is quantified based on the feedbacks collected from test-takers. Because the items are thus evaluated objectively, an item can be computed by

$$
\mathrm{DL}_{i}=\frac{\text { the number of correct answers }}{i},
$$

with $\mathrm{DL}_{i} \in(0,1)$; however, to assure the quality of questions in a bank, the questions' chosen difficulty values are in the range [0.1, 0.9].

When we extract $N$ tests, the tests must satisfy the constraints that are described as follows:

$C_{1}$ : The average difficulty level of questions in the exam must be equal to the difficulty level required from the user:

$$
\mathrm{DL}_{R}=\frac{\sum_{j=1}^{m} Q_{i j} \cdot \mathrm{DL}}{m} .
$$

$C_{2}$ : One ensures that the questions do not overlap with each other: $C_{i j}$ for each of the questions included in the test is thus unique; that is $\forall C_{i j} \in Q_{i}, \nexists C_{i j^{\prime}} \in Q_{i}: \Rightarrow$ $C_{i j} \cdot \mathrm{CP}=C_{i j^{\prime}} \cdot \mathrm{CP}$.

$C_{3}$ : This constraint ensures that each question's difficulty level, $Q_{i j} \cdot \mathrm{DL}$, is not the same as the required difficulty value (convergence on the required difficulty value) $\left(\mathrm{DL}_{R}\right): \forall C_{i j} \in Q_{i}, \nexists C_{i j^{\prime}} \in Q_{i} \Rightarrow C_{i j} \cdot \mathrm{DL}=C_{i j^{\prime}} \cdot \mathrm{DL}=\mathrm{DL}_{R}$.

$C_{4}$ : This constraint ensures that all the questions in one group must appear together. That means that if we pick up a single question out of a specific group then we must also take the rest of them. Suppose there is a group of questions $Q_{k}=\left\{Q_{k 1}, Q_{k 2}, Q_{k 3}, Q_{k 4}\right\}$, if $Q_{k j} \in Q_{i}$, then it is obligatory that $Q_{k} \in Q_{i}$. 
$C_{5}$ : This constraint ensures that the right number of questions exists in each section (CP). Assume the question bank includes $p$ sections, such as $P=$ $\left\{\mathrm{CP}_{1}, \mathrm{CP}_{2}, \mathrm{CP}_{3}, \mathrm{CP}_{4}, \ldots, \mathrm{CP}_{p}\right\}$, then it is necessary to extract $q$ sections $P_{i}=$ $\left\{\mathrm{CP}_{i 1}, \mathrm{CP}_{i 2}, \mathrm{CP}_{i 3}, \mathrm{CP}_{i 4}, \ldots, \mathrm{CP}_{i q}\right\}\left(P_{i} \subseteq P\right)$, where each section $\mathrm{CP}_{i j}$ has the required number of questions denoted by $\mathrm{NQ}_{R}$, which satisfies the condition: $\sum_{j=1}^{q} \mathrm{CP}_{i j} \cdot N Q_{R}=m$.

\subsection{Target function}

The objective function of the problem can be proposed as follows:

$$
f\left(Q_{i j} \cdot \mathrm{DL}\right)=\frac{\sum_{j=1}^{m} Q_{i j} \cdot \mathrm{DL}}{m}-\mathrm{DL}_{R} \rightarrow \min ,
$$

where $m$ is the total number of questions, $Q_{i j} \cdot$ DL is the difficulty of each question and $\mathrm{DL}_{R}$ is the difficulty-level requirement.

The objective function $f\left(Q_{i j} \cdot \mathrm{DL}\right)$ is used as the fitness function in the algorithm, and the results of the objective function are considered as the fitness of the resulting test.

In this case, the better the fitness, the smaller the $f\left(Q_{i j} \cdot \mathrm{DL}\right)$ becomes. To improve the quality of the test, we also take into account the constraints $C_{1}, C_{2}, C_{3}$, $C_{4}$ and $C_{5}$, as mentioned in Sec. 3.1.

\subsection{Particle swarm optimization to extract tests}

When using PSO to extract a test, the operators used in the proposed algorithm are as follows:

Step 1. Using random for initializing the population.

Step 2. Select $G_{\text {best }}$ and $P_{\text {best }}$.

Step 3. IF $G_{\text {best }}$ satisfies the stop conditions THEN exit.

Step 4. Update the locations of individuals.

Step 4.1. The $P_{\text {best }}$ individuals approach $G_{\text {best }}$, with $V_{P_{\text {best }}}$.

Step 4.2. $G_{\text {best }}$ approaches goal, with $V_{G_{\text {best }}}$.

Step 5. Go to Step 2 .

Here $f\left(Q_{i j} \cdot \mathrm{DL}\right)=\frac{\sum_{j=1}^{m} Q_{i j} \cdot \mathrm{DL}}{m}-\mathrm{DL}_{R}$ (in Sec. 3.1) is a fitness function, $P_{\text {best }}$ is the locally optimal solution, with $P_{\text {best }}=\min \left(f_{1}, f_{2}, \ldots, f_{i}\right)$ (where $f_{i}$ is the number of particles $), G_{\text {best }}$ is the globally optimal solution, with $G_{\text {best }}=\min \left(P_{\text {best } 1}\right.$, $\left.P_{\text {best }_{2}}, \ldots, P_{\text {best }_{i}}\right), V_{P_{\text {best }}}$ is the velocity of $P_{\text {best }}$, with $V_{P_{\text {best }}}=\alpha \times$ the total number of questions in the test, where $\alpha$ is a percentage defined by the user $(0<\alpha<1), V_{G_{\text {best }}}$ is the velocity of $G_{\text {best }}$, with $V_{G_{\text {best }}}=\beta \times$ the total number of questions in the test, where $\beta$ is a percentage defined by the user $(0<\beta<1)$. 
Working principle of multi-swarm particle swarm optimization for extracting a test is as follows:

- The Selection operator selects the global optimal $G_{\text {best }}$ and local optimal $P_{\text {best }}$ based on fitness. The best individual in a population is called $G_{\text {best }}$, and the others are called $P_{\text {best }}$.

- The $P_{\text {best }}$ individuals move to $G_{\text {best }}$ by receiving information about the location of $G_{\text {best }}$, information about the location of $G_{\text {best }}$ in the problem of extraction is the question. $P_{\text {best }}$ moves to $G_{\text {best }}$ with a velocity vector, which is the number of questions transferred from $G_{\text {best }}$ to $P_{\text {best }}$. After that, $P_{\text {best }}$ receives random questions from $G_{\text {best }}$, and the new location of $P_{\text {best }}$ is close to that of $G_{\text {best }}$. The fitness value is then checked, and if the fitness of the new location is better than that of the old location then the system updates $P_{\text {best }}$ based on the new location, otherwise, the old location is unchanged.

- Moving $G_{\text {best }}$ to the goal is not the same process as moving $P_{\text {best }}$ to $G_{\text {best }}$. In this process, $G_{\text {best }}$ replaces its question with other items from the question bank. The velocity vector is the number of questions as in the process when $P_{\text {best }}$ moves to $G_{\text {best }}$. After receiving questions and moving to a new position, if the fitness of $G_{\text {best }}$ at the new location is better than that of the old location, then the old location of $G_{\text {best }}$ is updated by the new location, otherwise, the old location is unchanged.

- Stop condition: The algorithm will stop when the fitness values are smaller than $\varepsilon$, with this being defined by the user.

\subsection{Multi-swarm particle swarm optimization for extracting tests}

As mentioned above, use of PSO-based method for extracting tests, in order to improve the convergence speed and diversity of extracting tests from question banks, often deals with very large search space. We present a novel method for the generation of multi-choice tests, which extracted an abundance of tests at the same time with equivalent levels of difficulty and approximated the specific difficulty-level requirement given by the user. The proposed approach for extracting tests from question banks is based on the parallelism of multi-swarm migration in the PSO algorithm. This approach is novel and better than the traditional approaches such as manually extracted tests, random methods, the original PSO algorithm, etc. in terms of the execution time and quality of tests.

Multi-swarm method used multi-threads to perform parallelism. Each thread corresponds to a thread running the PSO algorithm in Sec. 3.3. In this subsection, we present the parallel multi-swram single-objective particle swarm optimization (MSPSO) for increasing performance. Each swarm now corresponds to a thread and the information exchange happens by chance between swarms. The parallel MSPSO starts with locking the current thread (swarm) in order to avoid interference from other threads.

We Follow the theory of Lewis which describes a relationship between two regions, the subsistence sector and the capitalist sector. We can understand that the 
two types of economic sectors here are the strong (capitalist) sectors and the weak (subsistence) sectors while ignoring other aspects of the economy. Whether a sector is strong or weak depends on the fitness values of $G_{\text {best }}$ positions of its swarm. However, when applying those theories, some adjustments are made so that the parallel MSPSO can yield better optimal solutions. The direction of information exchange changes when individuals with strong $P_{\text {best }}$ (hereafter strong individuals) in strong sectors move to weak sectors. The weak sectors' $G_{\text {best }}$ may be replaced by the incoming $P_{\text {best }}$ and the fitness values of the weak swarms should make a large lift. The foremost condition for migration to happen is that there are changes in the fitness values of the current generations' $G_{\text {best }}$ compared to the previous generations' $G_{\text {best }}$.

The parallel MSPSO-based approach to extract multiple tests is described in a form of pseudocode in the following.

The process of generating multiple tests at the same time in a single run using parallel MSPSO includes two stages. The first stage is generating tests using singleobjective PSO. In this stage, the algorithm proceeds to find tests that satisfy the requirement and all the constraints using multiple threads. Each thread corresponds to each swarm that runs separately. The second stage is improving and diversifying tests. This stage happens when there is a change in the value of $G_{\text {best }}$ of each swarm (for each thread) in the first stage. In this second stage, migration happens between swarms to exchange information between running threads in order to improve the convergence and diversities of solutions.

\section{Experimental Studies}

The experimental environment and the description of experimental parameters are in Sec. 4.1; the evaluations on most criteria, such as execution time, search space, stability and standard deviation, are discussed in Secs. 4.2 and 4.3.

\subsection{Experimental environment}

Algorithms are implemented in C\# (Microsoft Visual Studio 2013), using Windows 8.1 Operating System, running on a computer with a 2.5-GHz CPU and 4-GB RAM. The experimental data includes a question bank with 1,200 different questions. The question bank also consists of multiple sections and each section has more than 150 questions with different difficulty levels as shown in Table 1. Experimental data of small question bank consists of 160 different questions and the question bank also consists of multiple sections and each section has more than 19 questions with different difficulty levels as shown in Table 2. The experimental parameters of MSPSO are presented in Table 3.

\subsection{Effect of the number of swarms (a number of tests are extracted)}

This experiment determines the effect of the number of swarms required for the extraction of the operational process of the algorithm. 
Table 1. Experimental data of large question bank.

\begin{tabular}{lccccccc}
\hline $\begin{array}{l}\text { Level of } \\
\text { difficulty }\end{array}$ & $\begin{array}{c}\text { Questions } \\
\text { part 1 }\end{array}$ & $\begin{array}{c}\text { Questions } \\
\text { part 2 }\end{array}$ & $\begin{array}{c}\text { Questions } \\
\text { part 3 }\end{array}$ & $\begin{array}{c}\text { Questions } \\
\text { part 4 }\end{array}$ & $\begin{array}{c}\text { Questions } \\
\text { part 5 }\end{array}$ & $\begin{array}{c}\text { Questions } \\
\text { part 6 }\end{array}$ & $\begin{array}{c}\text { Total } \\
\text { questions }\end{array}$ \\
\hline 0.2 & 30 & 40 & 28 & 23 & 33 & 21 & 175 \\
0.3 & 22 & 40 & 26 & 19 & 48 & 19 & 174 \\
0.4 & 24 & 40 & 19 & 22 & 26 & 19 & 150 \\
0.5 & 23 & 22 & 40 & 21 & 45 & 44 & 195 \\
0.6 & 23 & 22 & 22 & 17 & 28 & 44 & 156 \\
0.7 & 23 & 29 & 24 & 24 & 56 & 33 & 189 \\
0.8 & 27 & 19 & 16 & 49 & 26 & 24 & 161 \\
Total & 172 & 212 & 175 & 175 & 262 & 204 & 1,200 \\
\hline
\end{tabular}

Table 2. Experimental data of small question bank.

\begin{tabular}{lccccccc}
\hline $\begin{array}{l}\text { Level of } \\
\text { difficulty }\end{array}$ & $\begin{array}{c}\text { Questions } \\
\text { part 1 }\end{array}$ & $\begin{array}{c}\text { Questions } \\
\text { part 2 }\end{array}$ & $\begin{array}{c}\text { Questions } \\
\text { part 3 }\end{array}$ & $\begin{array}{c}\text { Questions } \\
\text { part 4 }\end{array}$ & $\begin{array}{c}\text { Questions } \\
\text { part 5 }\end{array}$ & $\begin{array}{c}\text { Questions } \\
\text { part 6 }\end{array}$ & $\begin{array}{c}\text { Total } \\
\text { questions }\end{array}$ \\
\hline 0.2 & 4 & 3 & 3 & 5 & 3 & 7 & 25 \\
0.3 & 2 & 5 & 5 & 1 & 7 & 2 & 22 \\
0.4 & 5 & 5 & 5 & 2 & 4 & 8 & 29 \\
0.5 & 1 & 5 & 5 & 3 & 4 & 4 & 22 \\
0.6 & 6 & 1 & 0 & 2 & 4 & 3 & 16 \\
0.7 & 1 & 7 & 3 & 2 & 1 & 2 & 16 \\
0.8 & 2 & 2 & 4 & 4 & 5 & 3 & 20 \\
Total & 21 & 28 & 25 & 19 & 28 & 29 & 150 \\
\hline
\end{tabular}

\subsubsection{Large question bank in Table 1}

We use the parameters in column (2) in Table 3. The results are shown in Fig. 1.

The processing time of the migration-based multi-swarm method is always less than the nonmigration-based method; the difference is more noticeable when there is an increase in the number of swarms in the algorithm. In particular, the number of generations in the migration-based method decreases as the number of swarms increases without any decline in the processing time. As the standard deviation is zero, it shows that increasing the number of swarms in the algorithm does not decrease the quality of the search results for the bank with a large number of questions (12,000 questions).

\subsubsection{Small question bank in Table 2}

We use the parameters in column (3) in Table 3. The results are shown in Fig. 2.

We find that the processing time increases as the number of swarms increases, the standard deviation of both methods is unaffected by changing the swarm numbers. However, with question banks that are small, we can see a difference in search capability of the two methods. The processing time of the migration-based method is 


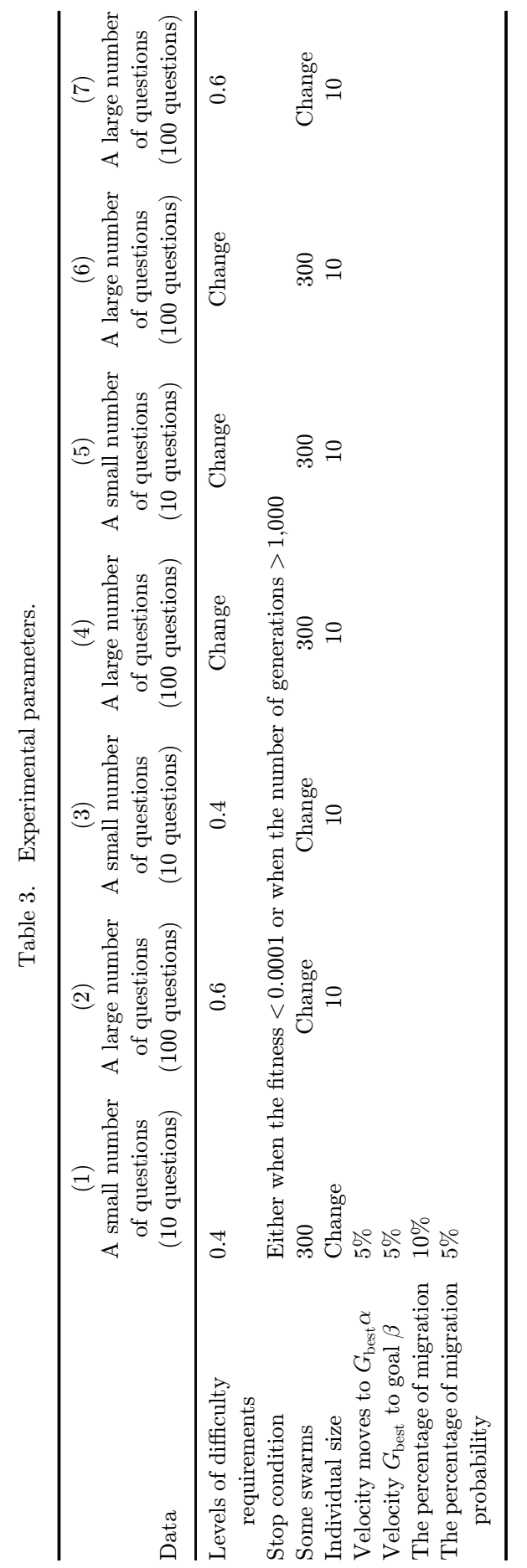




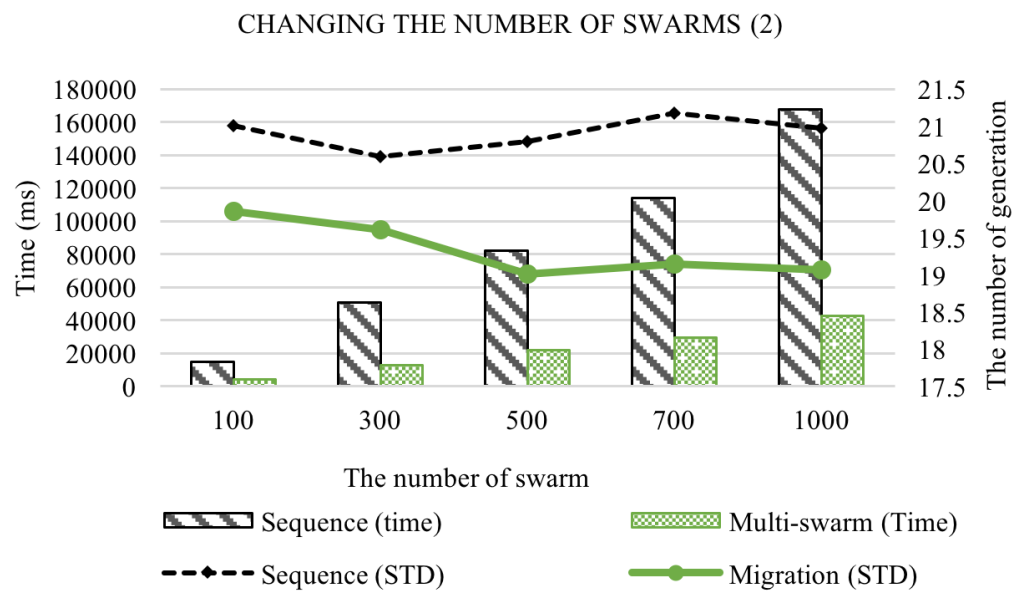

Fig. 1. Changing the number of swarms with parameters in Table 3, column (2).

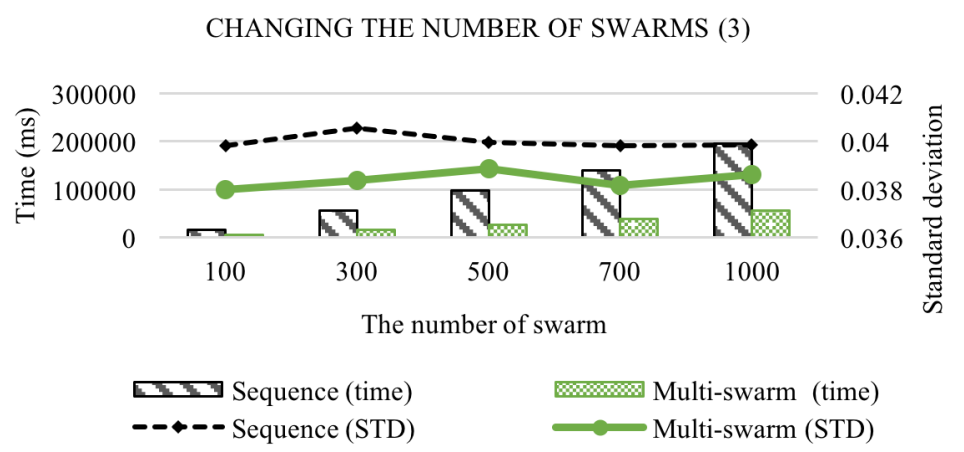

Fig. 2. Changing the number of swarms with the parameters in Table 3, column (3).

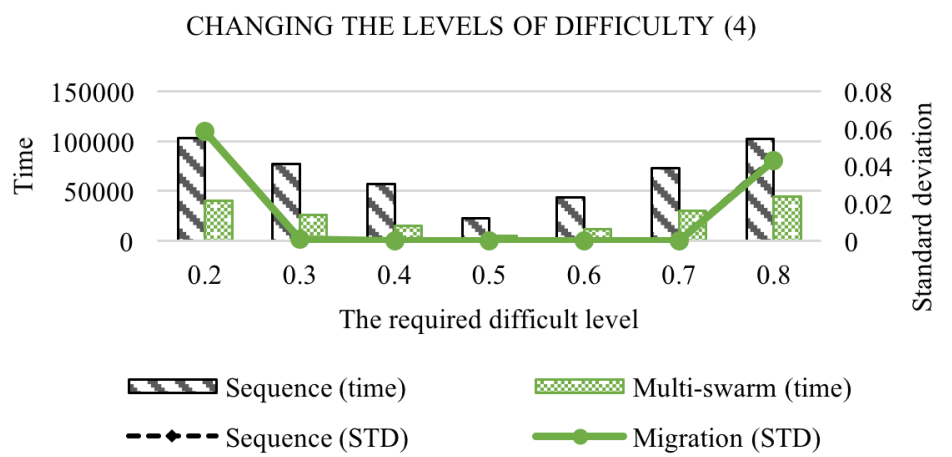

Fig. 3. Changing the levels of difficulty with the parameters in Table 3, column (4). 


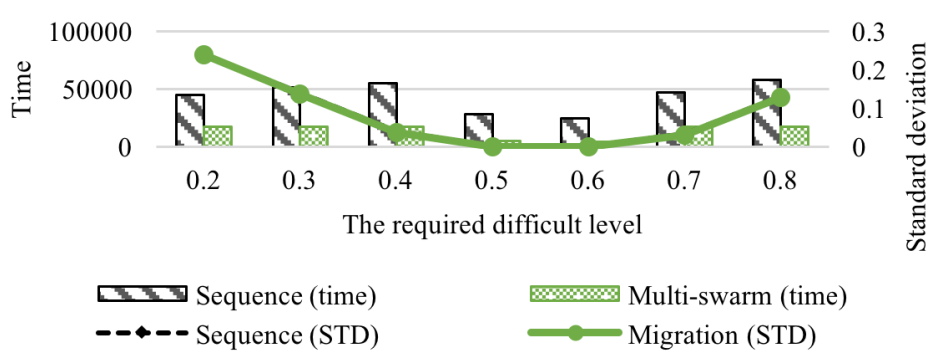

Fig. 4. Changing the levels of difficulty with the parameters in Table 3, column (5).

always better than the nonmigration-based one; the standard deviation is significantly improved (about 50\% on average) with better search results than the old nonmoving method.

\subsection{Evaluation of the difficulty-level requirements}

This experiment aims to evaluate the effect of difficulty-level requirements of the algorithm.

\subsubsection{Large question bank in Table 1}

We use the parameters in column (4) in Table 3. The results are shown in Fig. 3.

Based on the experimental results, we could have a general idea about when difficulty-level requirements change will affect the search capability of the algorithm. It can be seen that the closer the difficulty level to the margins, the more difficult it is to extract the tests, so processing time for generation and the standard deviation are longer. However, the migration-based multi-swarm methods demonstrate that their processing speed, generation and standard deviation are better than nonmigrationbased methods on every different difficulty-level requirement.

\subsubsection{Small question bank in Table 2}

We use the parameters in column (5) in Table 3. The results are shown in Fig. 4.

Because the sizes of the question banks are small, the processing time is short, and the number of generations is almost the same. While the difficulty-level requirements range between 0.2 and 0.8 , migration-based methods also show faster processing time than nonmigration-based methods. Especially at the difficulty levels of 0.5 and 0.6 , both algorithms find the correct solution to the problem, which again proves that the difficulty-level requirements though varying in values always affect the search capability of the algorithms. This effect clearly can be seen when we compare the standard deviations at closer values to the margins and at the low-to-mid range at the difficulty-level requirements of 0.5 and 0.6. 


\section{Conclusions and Future Studies}

In this paper, we present an approach to extract an abundance of tests with equivalent levels of difficulty and approximate the specific difficulty-level requirement given by the user based on question banks and the parallelism of multi-swarm method in PSO algorithm. This approach is novel and performs better than the traditional techniques such as manually extracted of tests, random methods, etc. in terms of the execution time and the quality of tests. The experimental results demonstrated that the developed algorithm produces solutions of good quality (viz. optimal and near-optimal solutions) in a short amount of computing time.

However, the study still has its limitations with regard to the diversity of the solutions during the extraction process, so we may apply some other migration theories to help diversify the solutions to a greater degree.

Future studies will focus on investigating the use of the proposed hybrid approach $^{12}$ to solve other NP-hard and combinatorial optimization problems, which are expected to improve the efficiency of the method for evaluating the difficulty level of each question and extracting the tests. Other relevant studies may also focus on fine-tuning the PSO parameters by using some type of adaptive strategies. Indeed, in this study, no in-depth attempt was done to optimize the running parameters for PSO, which could improve the performance of the proposed approach.

\section{References}

1. M. Yildirim, Heuristic optimization methods for generating test from a question bank, in MICAI 200\%: Advances in Artifical Intelligence, Lecture Note in Computer Science, Vol. 4827 (Springer, Berlin, 2007), pp. 1218-1229.

2. M. Yildirim, A genetic algorithm for generating test from a question bank, Comput. Appl. Eng. Educ. 18(2) (2010) 298-305.

3. T. Bui, T. Nguyen, B. Vo, T. Nguyen, W. Pedrycz and V. Snasel, Application of particle swarm optimization to create multiple-choice tests, J. Inf. Sci. Eng. 34(6) (2018) $1405-1423$.

4. J. Kennedy and R. C. Eberhart, Particle swarm optimization, in Proc. IEEE Int. Conf. Neural Networks (IEEE, Piscataway, 1995), pp. 1942-1948.

5. H. Zhou, M. Song and W. Pedrycz, A comparative study of improved GA and PSO in solving multiple traveling salesmen problem, Appl. Soft Comput. 64 (2018) 564-580.

6. S. Shabir and R. Singla, A comparative study of genetic algorithm and the particle swarm optimization, Int. J. Electr. Eng. 9(2) (2016) 215-223.

7. P. D. Sheth and A. J. Umbarkar, Constrained optimization problems solving using evolutionary algorithms: A review, in Proc. Int. Conf. Computational Intelligence and Communication Networks (2015), pp. 1251-1257.

8. B. Niu, Y. Zhu, X. He and H. Wu, MCPSO: A multi-swarm cooperative particle swarm optimizer, Appl. Math. Comput. 185 (2007) 1050-1062.

9. D. D. Patil and B. D. Dangewar, Multi-objective particle swarm optimization (MOPSO) based on Pareto dominance approach, Int. J. Comput. Appl. 107(4) (2014) 13-15.

10. Y. Zhou and Y. Tan, GPU-based parallel multi-objective particle swarm optimization, Int. J. Artif. Intell. 7(A11) (2011) 125-141. 
11. Y. Zhang, S. Wang and G. Ji, A comprehensive survey on particle swarm optimization algorithm and its applications, Math. Probl. Eng. 2015 (2015) 931256.

12. F. Zou, D. Chen, R. Lu and P. Wang, Hierarchical multi-swarm cooperative teachinglearning-based optimization for global optimization, Soft Comput. 21(23) (2017) 6983-7004.

13. D. Wang, D. Tan and L. Liu, Particle swarm optimization algorithm: An overview, Soft Comput. 22(2) (2018) 387-408. 